\title{
Microcontroller Enabled Portable Device for Flow Measurement of a Conducting Liquid
}

\author{
Saffna Shajahan ${ }^{1}$, Jyothi Krishna ${ }^{2}$,Linsa Hussain ${ }^{3}$ \\ ${ }^{1,2,3}$ (M.Tech. Scholar, Applied Electronics and Instrumentation, LMCST, University of Kerala, India
}

\begin{abstract}
The accurate measurement of gas and liquid is important to obtain specific proportions as per process requirement is necessary. The maintenance of definite rates of flow is important for maximum efficiency and production. The paper proposes a novel technique for the measurement of flow of a conducting liquid in which no excitation coil is required. The technique consists of four metal electrodes inserted into the selected zone of the flow tube, which are always in contact with the liquid and produce no obstruction to liquid flow. This electrode system is excited from an ac source, and the output signal has been found to be linearly related with the liquid flow under the laminar-flow condition, along with a small nonlinear zone at turbulent conditions. Index Terms: Metal Electrode system, Microcontroller, Wien bridge oscillator.
\end{abstract}

\section{INTRODUCTION}

Measurement of flow rate and quantity is the oldest of all measurements of process variables in the field of instrumentation. It is made for determining the proportions and the amount of materials flowing in or out of a process. For the purpose of cost accounting, such measurement is often required to be made for steam, water and gas services. Without flow measurements, plant material balancing, quality control and even the operation of any continuous process would be almost impossible. Nearly all of the products manufactured by industry are influenced in some way by the flow of materials. This type of processing ranges from the simple flow of fuel or gas in a heat-treating furnace to the control of large amounts of oil or gas passing through a pipeline. The measurement and control of flow variables is one of the most important process control application of industry.

\section{SYSTEM OVERVIEW}

The block diagram discusses briefly the basic building blocks involved for measuring the flow of a conducting liquid. The metal electrode system of four electrodes form a lumped-parameter bridge circuit and is supplied from a stabilized Wien bridge oscillator at $5 \mathrm{~V}$ and $1000 \mathrm{~Hz}$. The four metal electrodes following the Wheatstone bridge network principle are inserted into the selected zone of the flow tube, which are always in contact with the liquid and produce no obstruction to liquid flow

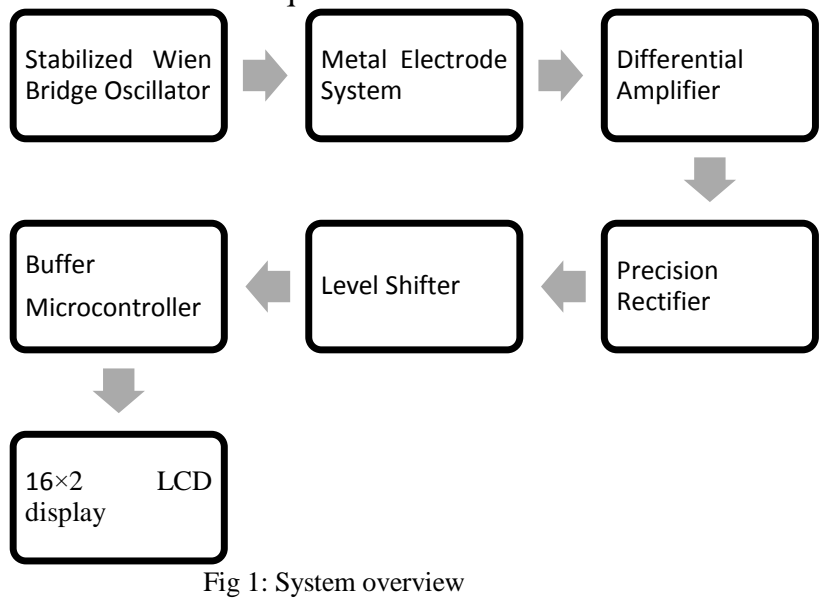

Any two diagonal electrodes are taken as the bridge supply nodal points, and the other two diagonal electrodes are taken as the bridge output nodal points. The bridge output voltage signal is first amplified by a differential amplifier. The amplified ac signal is converted to dc signal by a precision rectifier. The signal is then further amplified to a voltage signal in the range of $1-3.3 \mathrm{Vby}$ a level shifter. The offset or zero-adjustment network controls the offset of the rectified signal. The zero-offset adjustment is obtained by a buffer. The output of buffer is input to the microcontroller through an in-built analog-to-digital converter. The digital output of the microcontroller is displayed using a $16 \times 2 \mathrm{LCD}$ display. 


\section{SYSTEM DESCRIPTION}

\section{A. Wien bridge oscillator}

A Wien bridge oscillator is a type of electronic oscillator that generates sine waves. It can generate a large range of frequencies. The circuit is based on an electrical network originally developed by Max Wien in 1891. The bridge comprises four resistors and two capacitors. It can also be viewed as a positive feedback system combined with a band pass filter. The Wien bridge oscillator supplies the ac source at $5 \mathrm{~V}, 1000 \mathrm{~Hz}$ to the metal electrode system. It produces a very low distortion sine wave by using two RC networks connected to the positive terminal to form a frequency selective network. It causes oscillations to occur and amplifies the signal with two negative feedback resistors.

\section{B. Metal electrode system}

When two metal electrodes are immersed in a conducting liquid, the polarization effect at each electrode produces an electric double layer at each metal liquid interface, across of which there exists a difference in potential along with polarization impedance. If a very small value of sinusoidal voltage signal is applied across the electrodes, then this ac voltage signal is superimposed with the half-cell potential at each electrode, and accordingly, the flow of ions from one electrode to the other is obtained. The electric current due to this flow of ions may be opposed by the polarization impedance and the ohmic impedance of the liquid between the two electrodes. Again, if there is a flow of liquid from one electrode to the other, then the flow of liquid elements along with the ions may be opposed by the viscous effect of the liquid produced by the velocity gradient. Hence, the net impedance between the electrodes defined as the ratio of the supply voltage to the current may be assumed to be dependent on the electrode polarization effect, the ohmic effect, and the velocity gradient of the liquid. The polarization effect on linearly depends on the electric current density and the frequency of excitation, and the ohmic effect may be assumed constant for a particular system. When liquid flows through a pipeline, there exists a velocity gradient across the cross section of the pipeline, which depends on the flow rate of the liquid if the polarization and ohmic effects are assumed to be constant.

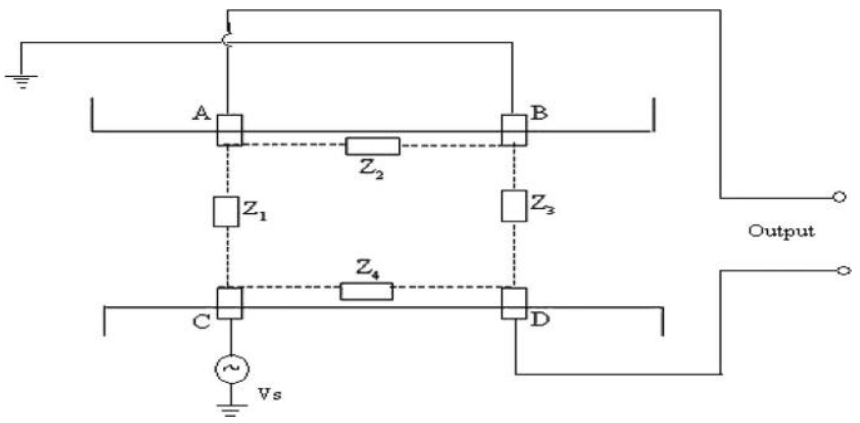

Fig2. Lumped-parameter equivalent impedance network between four electrodes.

\section{Differential Amplifier}

The input to differential amplifier is a pair of input pins, neither of which is connected to ground. Such pair of "floating" input connections is termed "differential inputs". Each input connection drives separate, balanced transistor amplifiers. Ideally, the same voltage connected to each input would produce no net result; hence the operational amplifier would only detect the difference in the two input voltages. The voltage that both inputs experience is known as the common mode voltage. In practice, the voltages on each input are amplified separately and, due to component variations, never perfectly balance out to zero.

\section{Precision Rectifier}

The input op-amp U1 once again has its positive input connected to the circuit input so that its output voltage will be the same polarity as the input voltage. Resistances R1 and R4 provide a path to ground for input bias currents as. For positive input voltages, U1's output is also positive. The output is connected through diode D2 to the non inverting input of op-amp U2. U2's output also rises until its positive and negative inputs are at the same voltage. At this point, the negative inputs of $\mathrm{U} 1$ and $\mathrm{U} 2$ are at the same because very little current is flowing through R2 or diode D1. The output of U1 continues to rise until its positive and negative inputs are equal. The output of $\mathrm{U} 2$ follows the input signal, supplying the positive half-cycle to the circuit output. For negative input voltages, U1's output is also negative, reverse biasing D2 so it acts as an open circuit. U1's negative output pulls current through D1 and R2. U2's output rises until the current flowing in R3 exactly balances the current flowing in R2. If R2 and R3 are equal and their currents are equal, the voltage at the output of $\mathrm{U} 2$ will be equal and opposite polarity to the voltage at the negative input of U1. U1's output raises the 
current through D1 and R2 until the voltage at its negative input equals the input voltage. So the output of U2 is also positive for negative input signals as D1 and D2 switch the current paths.

\section{E. Level shifter}

In applications requiring a unipolar AC voltage signal, the signal may be delivered from a bi-polar differential amplifier and repositioned relative to a zero volt reference (an earth ground) using a voltage level shifting circuit. In addition, the voltage level shifting technique is frequently used to generate high voltage DC outputs from lower voltage AC sources. The maximum voltage (AC or DC) produced at the output of a single stage of the level shifting circuit is nominally equal to the peak-to-peak value of the AC signal delivered by the differential amplifier.

\section{F.Buffer}

Due to the strong (i.e., unity gain) feedback and certain non-ideal characteristics of real operational amplifiers, this feedback system is prone to have poor stability margins. Consequently, the system may be unstable when connected to sufficiently capacitive loads. In these cases, a lag compensation network can be used to restore stability. Unity gain circuits are used as electrical buffers to isolate circuits or devices from one another, loading effects and prevent undesired interaction. As a voltage following power amplifier, this circuit will allow a source with low current capabilities to drive a heavy load. The gain of the voltage follower with the feedback loop closed (closed loop gain) is unity. The gain of the ideal operational amplifier without a feedback loop (open loop gain) is infinity. Thus, we have traded gain for control by adding feedback.

\section{G. PIC1 6F73 Microcontroller}

This powerful (200 nanosecond instruction execution) yet easy-to-program (only 35 single word instructions) CMOS FLASH-based 8-bit microcontroller packs Microchip's powerful PIC® architecture into 28pin package and is upwards compatible with the PIC16C5X, PIC12CXXX and PIC16C7X devices. The PIC16F73 features 5 channels of 8-bit Analog-to-Digital (A/D) converter with 2 additional timers, 2 capture/compare/PWM functions and the synchronous serial port can be configured as either 3-wire Serial Peripheral Interface (SPI $\left.{ }^{\mathrm{TM}}\right)$ or the 2-wire Inter-Integrated Circuit $\left(\mathrm{I}^{2} \mathrm{C}^{\mathrm{TM}}\right)$ bus and a Universal Asynchronous Receiver Transmitter (USART). All of these features make it ideal for more advanced level A/D applications in automotive, industrial, appliances and consumer applications.

\section{H. 16×2LCD DISPLAY}

The 2 X16 Parallel LCD is an 8 bit or 4 bit parallel interfaced LCD. This unit allows the user to display text, numerical data and custom created characters. The LCD uses the HD44780 series LCD driver from Hitachi, or equivalent controller. The LCD is connected to a female 14-pin connector for easy interface with the BS2p24/40 Demo Board (\#45187) and the Professional Development Board (\#28138). Though the device has the ribbon cable and 14-pin connector it may also be hooked up manually

\section{DESIGN AND EXPERIMENTAL SETUP}

The design of the flow head is very simple, as shown in fig 3. It only involves the selection of the electrode material, which is chemically inert to the flowing liquid. In the present design, the flow rate of water through a 1-in pipeline has been measured. Hence, the electrode material is selected to be stainless steel. Since the outside and inside diameters of the pipe are 25 and $20 \mathrm{~mm}$, respectively, the length of each electrode is selected to be $15 \mathrm{~mm}$ with 2-mm insertion depth. The diameter of each electrode is selected to be $4 \mathrm{~mm}$. These selections are made on a trial basis so that the obstruction to the flow is minimum. Each electrode is covered with a polyvinyl chloride sleeve and is fitted with a $2-\mathrm{mm}$ terminal screw. Each electrode is then mounted on the flow head along its line of symmetry through a proper hole so that the electrode may be inserted with a tight shut-up condition, and there is no water leakage at the operating pressure. The small gap is sealed with araldite. The length of the flow head is selected to be $3 \mathrm{ft}$, with the flange connections at both ends of the internal diameter being exactly equal to that of the pipeline. The flow head is connected with the pipeline through an insulating gasket of Teflon sheet.

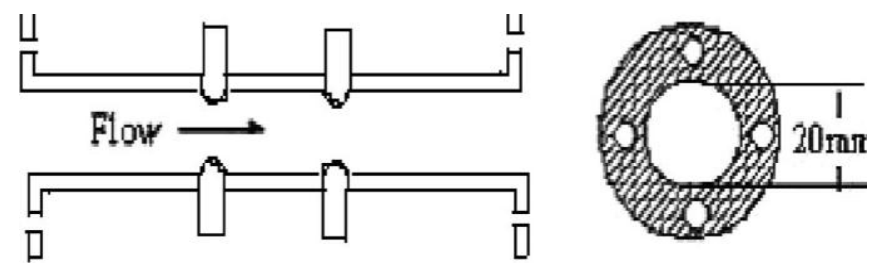

Fig 3. Flow head of bridge flow meter 
The experiment is performed by using tap water with the experimental setup as shown in Fig. 4. During the experiment, the inlet flow rate of water from the tap valve for a rotation of 360 degrees is converted into 36 degree for every $10 \%$ and a set of five readings are taken till $100 \%$. The flow can be maintained at a constant value using each set of observations. Hence, accurate calibration of the flow head is possible .After this adjustment, the water from the tap is made to flow through the pipeline was increased in small steps, each step corresponding to $10 \%$ of valve opening. In each step the signal conditioner output were noted in both increasing and decreasing modes for five sets of experimental data. The experimental data are tabulated in Table 3.1. The static calibration graphs thus obtained for the experiment from the signal conditioner output are shown in Results. The standard deviation curve for five sets of experimental data in both increasing and decreasing modes is drawn.

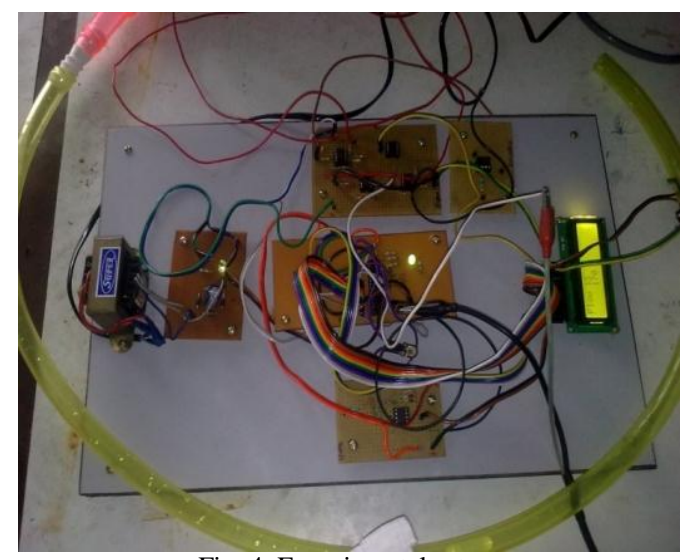

Fig 4. Experimental set up

\section{RESULT AND CONCLUSION}

The repeatability of the experimental data in the increasing and decreasing modes was found to be satisfactory, as shown by the standard deviation curve in Fig. 3.3. The calibration graphs shown in Fig. 5.1reveals that the nature of the graph is piecewise linear over a large portion of the operating zone. The nonlinearity may be due to the turbulence effect of flow. The nature of the graph appears to follow the theoretically derived equations [see (11) and (19)]. The technique is very simple and requires no magnetic excitation field coils, as in the case of an electromagnetic flowmeter, and hence, it has very low cost. The experiment was conducted a t $30 \circ \mathrm{C}$ (room temperature), at which the kinematics viscosity of water is $v=0.801$ $\times 10-6 \mathrm{~m} 2 / \mathrm{s}$. Again, the limiting value [17], [18] of Reynolds's number $[\operatorname{Re}=(V \times d) / v$, where $V$ is the velocity of the liquid, and $d$ is the internal diameter of the pipe] for laminar flow is $R e<2100$, that for transient flow is $2300<R e<4000$, and that for turbulent flow is $R e>4000$. Thus, for a pipe inside diameter $(d)$ of $0.02 \mathrm{~m}$, the maximum flow rate under laminar conditions at $R e<2100$ is $1.6 \mathrm{~L} / \mathrm{min}$, and the flow rate under turbulent conditions at $R e>4000$ is $3.02 \mathrm{~L} / \mathrm{min}$.

TABLE1 : Experimental Result Data

\begin{tabular}{|c|c|c|c|c|c|c|c|c|}
\hline \multirow{2}{*}{$\begin{array}{r}\text { S1 No: } \\
1\end{array}$} & \multirow{2}{*}{\begin{tabular}{|}
$\begin{array}{c}\text { Valve } \\
\text { Opening } \\
\text { in \% }\end{array}$ \\
10
\end{tabular}} & \multicolumn{5}{|c|}{$\begin{array}{l}\text { Signal conditioner ouput in Volts for five sets of } \\
\text { reading. }\end{array}$} & \multirow{2}{*}{\begin{tabular}{l|} 
Mean \\
.025 \\
\end{tabular}} & \multirow{2}{*}{$\begin{array}{c}\text { SD } \\
0.000002\end{array}$} \\
\hline & & 0.02 & 0.025 & 0.03 & 0.02 & 0.03 & & \\
\hline 2 & 20 & 0.02 & 0.03 & 0.03 & 0.02 & 0.03 & .026 & 0.000024 \\
\hline 3 & 30 & 0.03 & 0.03 & 0.025 & 0.02 & 0.035 & .028 & 0.000026 \\
\hline 4 & 40 & 0.035 & 0.03 & 0.035 & 0.04 & 0.03 & .034 & 0.000014 \\
\hline 5 & 50 & 0.04 & 0.04 & 0.045 & 0.05 & 0.04 & .043 & 0.000016 \\
\hline 6 & 60 & 0.04 & 0.04 & 0.05 & 0.05 & 0.05 & .046 & 0.000024 \\
\hline 7 & 70 & 0.05 & 0.05 & 0.045 & 0.05 & 0.04 & .047 & 0.000016 \\
\hline 8 & 80 & 0.06 & 0.05 & 0.04 & 0.05 & 0.04 & .048 & 0.000056 \\
\hline 9 & 90 & 0.06 & 0.05 & 0.07 & 0.06 & 0.05 & .058 & 0.000056 \\
\hline 10 & 100 & 0.07 & 0.07 & 0.06 & 0.07 & 0.05 & .064 & 0.000064 \\
\hline
\end{tabular}

The experimental graph, as shown in Fig. 5, also conforms to these data. Thus, it may be assumed that the flow under laminar conditions appears to follow (11) and that under turbulent conditions, it appears to follow (19). However, under higher turbulent conditions at a high flow rate of the liquid, the nonlinear equation (19) may include higher order terms, and the nonlinearity thus produced may be linearized by assuming more linear parts 
of the experimental graphs. In the electromagnetic flowmeter, the induced voltage across the sensing electrode due to the flow of a conducting liquid through an intense magnetic field is very small, and special care must be taken to reduce the effect of electromagnetic interference and the fluctuation of the supply voltage of the electromagnet. However, in the case of the proposed technique, the measurement may be assumed to be free from these effects. Since the effect of electromagnetic interference is identical to all the electrodes, it has negligible effect on the bridge output, and this effect is further reduced by a high common-mode rejection ratio of the instrumentation amplifier. Moreover, a bridge is supplied from a stabilized sinusoidal ac source, and hence, the effect of supply voltage fluctuation may be reduced by a proper design of the bridge supply source. The personnel error is minimized by repeating the same reading several times and taking the average. Another error may be due to the fluctuations of flow rate during the period of collection of water, which is minimized by keeping the valve head constant. Hence, the absolute error of measurement may be assumed to be negligible.

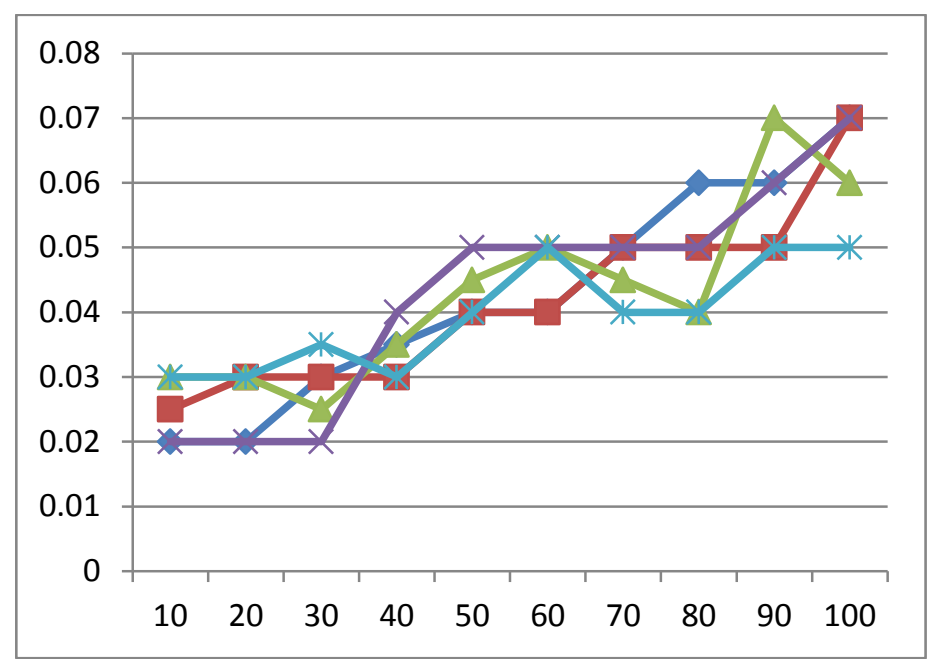

Fig. 5.1 Calibration Curve 1 (Valve Opening in \% Vs Signal Conditioner Output in Volts for 5 sets)

\section{REFERENCES}

[1]. B. Onaral and H.P. Schwan, "Linear and non-linear properties of platinum electrode polarization, Part I: Frequency dependence at very low frequent-cies,” Med. Biol, Eng. Comput., vol. 20, no.3, pp. 299-306, May 1982.

[2]. B. Onaral and H.P. Schwan, "Linear and non-linear properties of platinum electrode polarization, Part II: A Time domain analysis," Med. Biol.Eng. Comput., vol.21, no.2, pp. 210, Mar. 1983.

[3]. Dale R. Patieck and Stephen W. Fardo, "Industrial Process Control Systems.

[4]. D.M. Considine, Process Instruments and Control Hand Book, $2^{\text {nd }}$ ed. New York: McGraw-Hill, 1974.

[5]. E.H. Lewitt, "Hydraulics and Fluid Mechanics", $10^{\text {th }}$ ed. London, U.K.: Pitman.

[6]. E.O. Doeblin, Measurement System Application and Design, $4^{\text {th }}$ ed. New York: McGraw-Hill, 1990.

[7]. H.H. Sun and B. Onaral, "A unified approach to represent neutral electrode polarization," IEEE Trans, Biomed, Eng., vol. BME-30 no.7, pp. 399-406, Jul, 1983.

[8]. P.C. Rakhshit, Physical Chemistry, $3^{\text {rd }}$ ed. Sci. Book Agency, 1973, pp. 627-662.

[9]. R.W. Simpson, J.G. Berberian, and H.P. Schwan, "Non-linear AC and DC polarization of platinum electrodes," IEEE Trans. Biomed.Eng., vol. BME-27, no.3,pp. 166-171, Mar.1980.

[10]. Satish Chandra Bera and Badal Chakraborty, "A Nover Technique of Flow Measurement for a Conducting Liquid". IEEE Trans. Instrumentation and Measurement, vol. 58, No. 8, August 2009. 